\title{
Quality Evaluation of Wind Turbine Roller Bearing Profile in the Ultra-long Flexible Blade
}

\author{
Mingshu $\mathrm{Li}^{1}$, Yuhong Sun ${ }^{1,}$, Ling Wang ${ }^{2}$ and Zhiteng $\mathrm{Gao}^{3}$ \\ ${ }^{1}$ Department of Business Administration, Guangzhou College of Technology and Business, Guangzhou, 528138, China; \\ ${ }^{2}$ Changzhou University Huaide College, Jingjiang, 214500, China; \\ ${ }^{3}$ Jiangsu Key Laboratory of Hi-Tech Research for Wind Turbine Design, Nanjing University of Aeronautics and Astronautics, Nanjing \\ 210016, China.
}

\begin{abstract}
In order to accurately measure and evaluate the quality of the roller profile of the ultra-long flexible blade bearing, an error evaluation model is established for the arc segment and the straight segment of the roller based on the least square method, and then an overall quality evaluation model is proposed based on these two error models. Through the simulation of a standard wind turbine cylindrical roller bearing, it is found that the quality evaluation model established in this work can effectively measure and evaluate the contour line of the wind turbine bearing's roller. The overall absolute error is $0.0319 \mathrm{~mm}$, which is consistent with the set random error. The overall quality evaluation model is also valid for other types of bearings commonly used in the wind turbine, which include arc and straight segments, and can be used to evaluate the error quality of the roller profile of wind turbine bearings.
\end{abstract}

\section{Introduction}

With the enlargement of wind turbine diameter, the blades have developed from tens of meters to hundreds of meters. The lengthening of the blades increases the flexibility of the blades, and the weight of the nacelle. Then, the fluctuation of wind-turbine loads also puts stricter quality requirements on wind turbine bearing rollers [1-2]. It is becoming more and more important to accurately assess the quality of large wind turbine bearing rollers. Many scholars have evaluated the quality errors of the arc and straight sections of the bearing rollers, but there is a lack of systematic research on the overall quality evaluation model, and they rarely involve the fitting of the minimum overall error area of the multi-segment curve [3-5].

Therefore, based on the least square method of segmented fitting and multi-value optimization, for the ultra-long flexible wind turbine blade, the bearing error model is firstly established in segments, and then based on the error model, the overall quality evaluation model of the wind turbine bearing roller profile is proposed. Finally, the established evaluation model is used to simulate and analyse a set of bearing roller profiles, and analyse the applicability and effectiveness of the model.

\section{Mathematical model}

\subsection{Contour model of wind turbine bearing roller}

Generally, the hub line of the cylindrical roller of a wind turbine bearing (as shown in Fig.1) can be expressed by
Equation 1:

$$
\left\{\begin{array}{l}
\left(x-a_{1}\right)^{2}+\left(y-b_{1}\right)^{2}=R_{1}^{2}\left(x_{0} \leqslant x \leqslant x_{1}\right) \\
y=k x+e\left(x_{1} \leqslant x \leqslant x_{2}\right) \\
\left(x-a_{2}\right)^{2}+\left(x-b_{2}\right)^{2}=R_{2}^{2}\left(x_{2} \leqslant x \leqslant x_{3}\right)
\end{array}\right.
$$

where:

$$
\left\{\begin{array}{l}
k=\frac{b_{1}-b_{2}}{a_{1}-a_{2}} \\
e=\frac{\left(a_{2} b_{1}-a_{1} b_{2}\right) \pm R_{1} \sqrt{\left(a_{1}-a_{2}\right)^{2}+\left(b_{1}-b_{2}\right)^{2}}}{a_{2}-a_{1}}
\end{array}\right.
$$

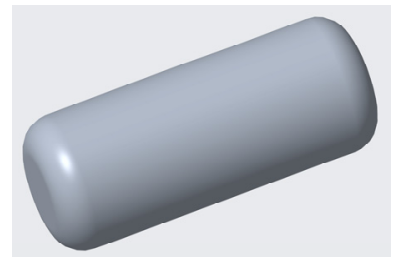

Fig. 1. Schematic of the cylindrical roller of wind power bearing

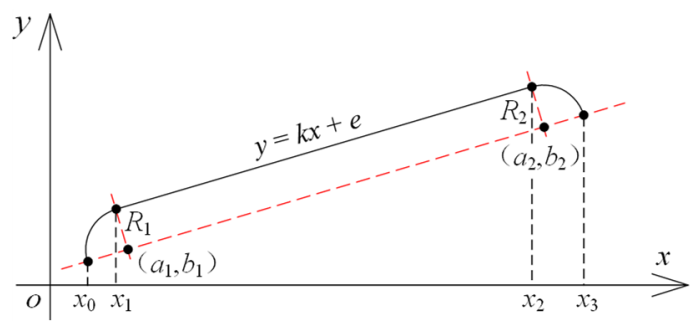

Fig. 2. Contour line of cylindrical roller

\footnotetext{
Corresponding author: sunyh0508@foxmail.com
} 


\subsection{Quality evaluation model}

The quality evaluation model is established according to the measurement error of the curve segment and the straight line segment. Because the error evaluation methods of the curve segment and the straight line segment are different, it is necessary to construct the quality evaluation model separately.

\subsubsection{Error model of arc segment}

Take $t$ measurement points around the critical points $P_{g}$ and $P_{h}$ as auxiliary critical points, and the area between them is the critical area, that is, the area where critical points may exist in the actual measurement. For the left arc, the left measurement point is used to form the fitting data with the right measurement point in turn, and the least square method is used for arc fitting. The left arc equation and the corresponding measurement error $E_{m}$ are as follows:

$$
\left\{\begin{array}{c}
\left(x-a_{m}\right)^{2}+\left(y-b_{m}\right)^{2}=R_{m}^{2} \\
E_{m}=\left|\Delta R_{m \max }\right|+\left|\Delta R_{m \min }\right|
\end{array}\right.
$$

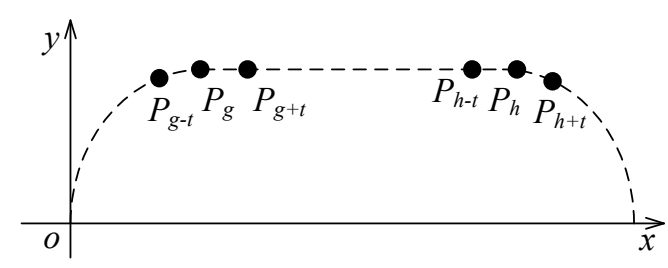

Fig. 3. Schematic of sampling points

In the equation: the arc deviation of the last measuring point of the arc $\Delta R_{m}=\sqrt{\left(x_{i}-a_{m}\right)^{2}+\left(y_{i}-b_{m}\right)^{2}}-R_{m}$. $\Delta R_{m \max }, \Delta R_{m \text { min }}$ are the largest circles respectively Arc deviation and minimum arc deviation. $m=1,2, \ldots, 2 t+1$, $\left(a_{m}, b_{m}\right), R_{m}$ are the coordinates and radius of the center point and radius of the fitted arc.

Similarly, for the right arc, the least square arc equation and the corresponding measurement error are as follows:

$$
\left\{\begin{array}{l}
\left(x-a_{n}\right)^{2}+\left(y-b_{n}\right)^{2}=R_{n}^{2} \\
E_{n}=\left|\Delta R_{n \max }\right|+\left|\Delta R_{n \text { min }}\right|
\end{array}\right.
$$

where: $n=1,2, \ldots, 2 t+1 . \Delta R_{n \max }, \Delta R_{n \min }$ are the maximum arc deviation and the minimum arc deviation respectively. $\left(a_{m}, b_{m}\right), R_{m}$ are the coordinates and radius of the center of the right arc, respectively.

\subsubsection{Linear error model}

Suppose the straight line equation of the middle straight line is $y=k x+e$, and the middle straight line is tangent to the two arcs. According to the constraint condition of the common tangent of the two arcs, it can be obtained:

$$
\left\{\begin{array}{l}
\left(2 k_{m n} e_{m n}-2 k_{m n} b_{m}-2 a_{m}\right)^{2}-4\left(k_{m n}^{2}+1\right) \times \\
\left(a_{m}^{2}+b_{m}^{2}-R_{m}^{2}+e_{m n}^{2}-2 b_{m} e_{m n}\right)=0 \\
\left(2 k_{m n} e_{m n}-2 k_{m n} b_{n}-2 a_{n}\right)^{2}-4\left(k_{m n}^{2}+1\right) \times \\
\left(a_{n}^{2}+b_{n}^{2}-R_{n}^{2}+e_{m n}^{2}-2 b_{n} e_{m n}\right)=0
\end{array}\right.
$$

By solving the above equations, and based on the principle of combining the tangent of the arc and the straight line, the equation of the common tangent of the $2 t$ +1 arcs at the left end and the $2 t+1$ arcs at the right end is $y=k_{m n} x+e_{m n}$, where $m=1,2, \ldots, 2 t+1, n=1,2, \ldots$, $2 t+1$, then the left and right tangent point coordinates are:

$$
\begin{aligned}
& \left(\begin{array}{l}
\frac{-\left(2 k_{m n} e_{m n}-2 k_{m n} b_{m}-2 a_{m}\right)}{2\left(k_{m n}^{2}+1\right)}, \\
\frac{-k_{m n}\left(2 k_{m n} e_{m n}-2 k_{m n} b_{m}-2 a_{m}\right)}{2\left(k_{m n}^{2}+1\right)}+e_{m n}
\end{array}\right) \\
& \left(\begin{array}{l}
\frac{-\left(2 k_{m n} e_{m n}-2 k_{m n} b_{n}-2 a_{n}\right)}{2\left(k_{m n}^{2}+1\right)}, \\
\frac{-k_{m n}\left(2 k_{m n} e_{m n}-2 k_{m n} b_{n}-2 a_{n}\right)}{2\left(k_{m n}^{2}+1\right)}+e_{m n}
\end{array}\right)
\end{aligned}
$$

Therefore, when the measurement point is located between the two tangent points, the measurement point participates in the calculation of the measurement error of the straight section; when it is outside the two tangent points, it participates in the calculation of the measurement error of the curve section. The distance from the measuring point to the common tangent is:

$$
D_{m n i}= \pm \frac{k_{m n} x_{i}+e_{m n}-y_{i}}{\sqrt{k_{m n}^{2}+1}}(u<i<v)
$$

In the Equation 8, the points involved in the calculation of the error of the straight line segment take a positive value on the upper part of the common tangent, otherwise take a negative value. The straightness error evaluation model is:

$$
E_{m n}=\max D_{m n i}-\min D_{m n i}
$$

\subsubsection{Overall quality evaluation model of cylindrical roller}

In the straightness error of the common tangent and the fitting error of the left and right arcs, the maximum error area is taken as the overall quality evaluation standard, and the maximum error value is recorded as $F_{m n}$. Therefore, the overall quality evaluation model of the cylindrical roller is:

$$
F=\min \left\{F_{m n}\right\}
$$




\section{Case study}

In order to evaluate the overall quality, a standard cylindrical roller bearing contour line is used as a mathematical model, a series of points are taken on the curve and random errors are introduced in the normal direction to form a series of simulation data points (Table 1), The absolute error value of random error is $0.02 \mathrm{~mm}$.

Table 1. Simulation data

\begin{tabular}{|c|c|c|c|c|c|}
\hline \multirow{2}{*}{ Index } & \multicolumn{2}{|c|}{$\begin{array}{c}\text { Point on curve } \\
(\mathbf{m m})\end{array}$} & $\begin{array}{c}\text { Normal } \\
\text { error } \\
(\mathbf{m m})\end{array}$ & \multicolumn{2}{c|}{$\begin{array}{c}\text { Simulation data } \\
(\mathbf{m m})\end{array}$} \\
\cline { 2 - 6 } & $x$ & $y$ & $\delta$ & $x_{i}$ & $y_{i}$ \\
\hline 1 & 0 & 0 & -0.016 & -0.016 & 0 \\
\hline 2 & 0.076 & 0.868 & 0.004 & 0.076 & 0.872 \\
\hline 3 & 0.302 & 1.710 & -0.010 & 0.300 & 1.700 \\
\hline 4 & 0.670 & 2.5 & 0.014 & 0.673 & 2.513 \\
\hline 5 & 1.170 & 3.214 & 0.014 & 1.175 & 3.227 \\
\hline 6 & 1.786 & 3.830 & 0.019 & 1.794 & 3.847 \\
\hline 7 & 2.5 & 4.330 & 0.000 & 2.500 & 4.330 \\
\hline$\ldots \ldots$ & $\ldots \ldots$ & $\ldots \ldots$ & $\ldots \ldots$ & $\ldots \ldots$ & $\ldots \ldots$ \\
\hline 38 & 32.5 & 4.33 & -0.012 & 32.488 & 4.329 \\
\hline 39 & 33.214 & 3.83 & -0.002 & 33.212 & 3.83 \\
\hline 40 & 33.83 & 3.214 & 0.018 & 33.848 & 3.216 \\
\hline 41 & 34.33 & 2.5 & -0.015 & 34.315 & 2.499 \\
\hline 42 & 34.699 & 1.71 & -0.001 & 34.697 & 1.71 \\
\hline 43 & 34.924 & 0.868 & 0.014 & 34.938 & 0.869 \\
\hline 44 & 35 & 0 & -0.018 & 34.982 & 0 \\
\hline
\end{tabular}

$$
\left\{\begin{array}{l}
(x-5)^{2}+y^{2}=25(0 \leqslant x<5) \\
y=5(5 \leqslant x<30) \\
(x-30)^{2}+y^{2}=25(30 \leqslant x<35)
\end{array}\right.
$$

Based on the equations in Section 2.1 and Section 2.2, the discrete data points in Table 1 is simulated and analyzed. The curvature and curvature difference of the discrete data points can be obtained, as shown in the Figure 4. The red point in Figure 4 is the minimum point of the curvature difference, and the extremum point can be selected to determine the adjacent region of the critical point. Therefore, the distribution of curvature difference can be used to determine the critical point, and the recognition efficiency is high.

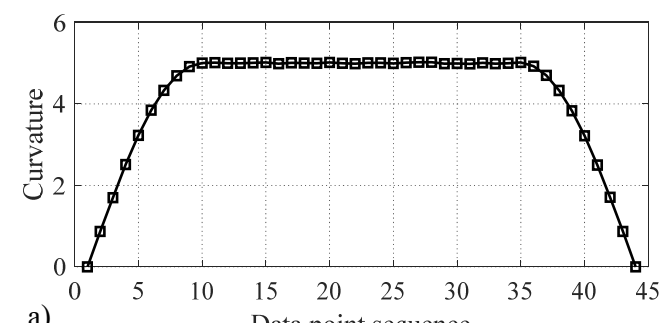

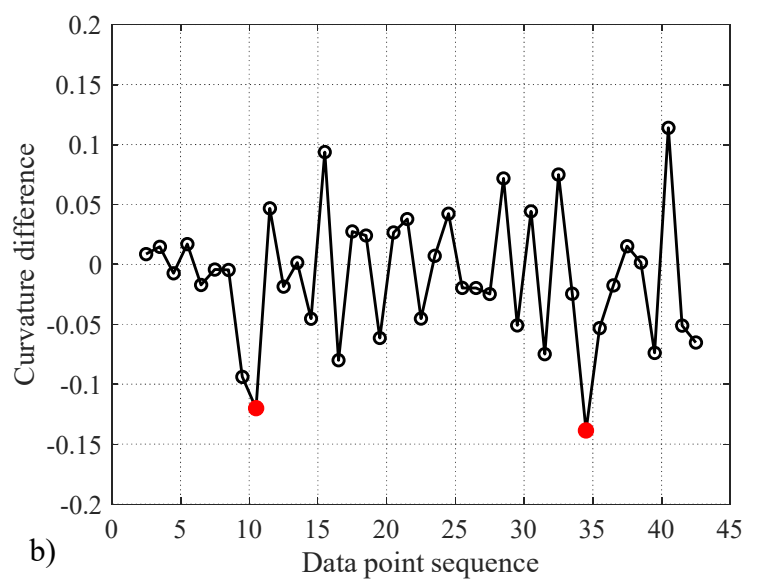

Fig. 4. Variation of curvature and curvature difference of data points

Take a measuring point on the left and right sides of the critical point as the auxiliary critical point, and use the least square method to fit the arc equation. The arc parameters and corresponding errors are shown in the following table:

Table 2. Left arc parameter and error analysis

\begin{tabular}{|c|c|c|c|c|}
\hline \multirow{2}{*}{$\begin{array}{c}\text { Data } \\
\text { point }\end{array}$} & \multicolumn{3}{|c|}{$\begin{array}{c}\text { Center point and radius of left } \\
\text { arc (mm) }\end{array}$} & $\begin{array}{c}\text { Error } \\
\text { (mm) }\end{array}$ \\
\cline { 2 - 5 } & $a$ & $b$ & $R$ & $E_{m}$ \\
\hline $\mathrm{P} 1 \sim \mathrm{P} 8$ & 4.9890 & -0.0046 & 4.9971 & 0.0159 \\
\hline $\mathrm{P} 1 \sim \mathrm{P} 9$ & 5.0011 & -0.0125 & 5.0104 & 0.0146 \\
\hline $\mathrm{P} 1 \sim \mathrm{P} 10$ & 5.1117 & -0.0962 & 5.1364 & 0.0845 \\
\hline
\end{tabular}

In the same way, the sampling data of the right arc and the middle straight section are constructed. According to Equation 10, the overall error of the wind turbine cylindrical bearing roller profile can be obtained as follows:

Table 3. Overall error of roller contour line of wind turbine cylindrical bearing

\begin{tabular}{|c|c|c|c|c|}
\hline Index & $\boldsymbol{E}_{\boldsymbol{m}}$ & $\boldsymbol{E}_{\boldsymbol{n}}$ & $\boldsymbol{E}_{\boldsymbol{m} \boldsymbol{n}}$ & $\boldsymbol{F}$ \\
\hline 1 & 0.0159 & 0.2231 & 0.1192 & 0.2231 \\
\hline 2 & 0.0159 & 0.0630 & 0.0510 & 0.0630 \\
\hline 3 & 0.0159 & 0.0319 & 0.0229 & 0.0319 \\
\hline 4 & 0.0146 & 0.2231 & 0.1160 & 0.2231 \\
\hline 5 & 0.0146 & 0.0630 & 0.0510 & 0.0630 \\
\hline 6 & 0.0146 & 0.0319 & 0.0234 & 0.0319 \\
\hline 7 & 0.0845 & 0.2231 & 0.0902 & 0.2231 \\
\hline 8 & 0.0845 & 0.0630 & 0.0389 & 0.0845 \\
\hline 9 & 0.0845 & 0.0319 & 0.0426 & 0.0845 \\
\hline
\end{tabular}

From the above table, the overall error of the sixth curve is the smallest, and the main parameters of the corresponding contour line are as follows:

Table 4. Comparison of set value and simulation result

\begin{tabular}{|c|c|c|c|c|c|c|c|c|c|}
\hline \multirow{3}{*}{ Index } & \multicolumn{8}{|c|}{ Main parameters of profile curve (mm) } & \multirow{3}{*}{$\begin{array}{c}\begin{array}{c}\text { Total error } \\
\text { (mm) }\end{array} \\
F\end{array}$} \\
\hline & \multicolumn{3}{|c|}{ Left arc radius and center } & \multicolumn{2}{|c|}{ Straight line parameters } & \multicolumn{3}{|c|}{ Right arc radius and center } & \\
\hline & $a$ & $b$ & $R$ & $k$ & $e$ & $a$ & $b$ & $R$ & \\
\hline Set value & 5 & 0 & 5 & 0 & 5 & 30 & 0 & 5 & 0 \\
\hline $\begin{array}{l}\text { Simulation } \\
\text { value }\end{array}$ & 4.989 & -0.005 & 4.997 & 0.0005 & 4.9898 & 29.992 & 0.0016 & 5.004 & 0.0319 \\
\hline
\end{tabular}


Table 4 shows that the left-end arc, right-end arc, and straight line parameters measured by this method are almost consistent with the set values, and the overall absolute error is $0.0319 \mathrm{~mm}$, which is consistent with the random error $0.02 \mathrm{~mm}$ introduced in this work. It can be seen that the error quality evaluation model established in this work can correctly evaluate the contour error of cylindrical roller bearings.

\section{Conclusion}

This work conducts error quality evaluation and analysis for cylindrical roller bearings commonly used in wind turbine yaw bearings. First, based on the least square method, the error models of the roller arc segment and the straight segment are established respectively. After that, an overall error evaluation model is established based on two types of error models. Finally, through a cylindrical roller bearing example, the difference between the set value of the roller bearing and the simulation result is analysed. The example analysis shows that the established roller bearing overall quality evaluation model can screen out the best contour curve, the simulation result is almost consistent with the set value, and the overall absolute error is consistent with the set random error. Therefore, the quality evaluation model proposed in this work can effectively measure and evaluate wind power cylindrical roller bearings. At the same time, for other tapered rollers, contact ball bearings and other types of bearing rollers that include arc segments and straight segments, the quality evaluation model proposed in this work is valid.

\section{Acknowledgment}

Thanks to Gansu Provincial Computing Center (GSPCC) for the computing resources contributed to this work. This research was funded by National Key Research and Development Project (No. 2019YFB1503700) and Priority Academic Program Development of Jiangsu Higher Education Institutions.

\section{References}

1. Gao Z, Wang T. Experimental validation and improvement of actuator line model in the large-eddy simulation of wind-turbine wakes[J]. IOP Conference Series: Earth and Environmental Science, 2020, 463(1): 012113.

2. Zheng Z, Gao Z, Li D, et al. Interaction between the atmospheric boundary layer and a standalone wind turbine in Gansu-Part II: Numerical analysis[J]. Science China Physics, Mechanics \& Astronomy, 2018, 61(09): 63-72.

3. Liu Z T, Yang J X, Zhao B. Study on Roundness Error Evaluation with Least-Squares Method Based on Nonlinear Optimization[J]. Advanced Materials Research, 2013, 765-767: 755-758.

4. $\mathrm{Gu}$ T, Lin S, Fang B, et al. An improved total least square calibration method for straightness error of coordinate measuring machine $[\mathrm{J}]$. Proceedings of the Institution of Mechanical Engineers Part B Journal of Engineering Manufacture, 2016, 230(9): 1665-1672.

5. Benko P, Kos G, Varady T. Constrained fitting in reverse engineering[J]. Computer Aided Geometric Design, 2002, 19(3): 173-205. 